\title{
On (3,3)-homogeneous Greechie Orthomodular Posets
}

\author{
Foat F. Sultanbekov ${ }^{1}$
}

\begin{abstract}
We describe (3,3)-homogeneous orthomodular posets for some cardinality of their sets of atoms. We examine a state space and a set of twovalued states of such logics. Particular homogeneous OMPs with exactly $k$ pure states $(k=1, \ldots, 7,10,11)$ have been constructed.
\end{abstract}

\section{INTRODUCTION}

Homogeneous orthomodular posets (OMPs) are important [10], [13]. They can be used in constructing counterexamples or OMPs with certain properties of the state space or the automorphisms group [5], [6], [7].

Let $n, m$ be natural numbers. An OMP, $L$, is called $(n, m)$-homogeneous $((n, m)$-hom. $)$, if its every atom is contained in $n$ maximal, with respect to inclusion, orhogonal sets of atoms (called blocks), and every such set of atoms of $L$ is $m$-element. The well known concrete logics of the form $\mathcal{L}_{q}^{p}=\{X \subset$ $\{1, \ldots, p q\} \mid \operatorname{card} X \equiv 0(\bmod q)\}[10]$ are nice examples of homogeneous OMPs. (3,3)-homogeneous logics arise when we consider relational OMPs [4] on a finite set. Orthomodular lattices of the kind were examined in [3],[12].

Let $L$ be a finite $(n, m)$-hom. OMP, $A$ the set of all atoms in $L, B$ the set of all blocks in $L, S=S(L)$ the set of all states on $L$, and $S_{2}$ the set of all two-valued states on $L$. A state $s$ on $L$ is called pure if $s$ is an extreme point of the convex set $S(L)$. It is easy to see that $n \cdot \operatorname{card} A=m \cdot \operatorname{card} B$.

Theorem 1.1.([10]) Suppose that $S_{2} \neq \emptyset$ and $f \in S_{2}$. Then $\operatorname{card} A=m k$, $\operatorname{card} B=n k$ where $k=\operatorname{card}\left(f^{-}(1) \cap A\right)$.

Let us recall some definitions of the theory of concrete logics [8], [9]. Let $\Omega$ be a set and $\mathcal{P}(\Omega)$ the Boolean algebra of all subsets of $\Omega$. A concrete logic (c.l.) on $\Omega$ is a subset $\mathcal{E}$ of $\mathcal{P}(\Omega)$ satisfying (1) $\Omega \in \mathcal{E} ; \quad(2) x \in \mathcal{E} \Rightarrow \Omega \backslash x \in$ $\mathcal{E} ; \quad(3) x, y \in \mathcal{E}, x \cap y=\emptyset \Rightarrow x \cup y \in \mathcal{E}$.

Denote by $V(\mathcal{E})$ the real vector space of all signed measures on $\mathcal{E}$ and put $\mathcal{E}^{\circ}=\{\mu \in V(\mathcal{P}(\Omega)) \mid \forall x \in \mathcal{E}(\mu(x)=0)\} . \mathcal{E}$ is called regular if every signed measure on $\mathcal{E}$ extends to a signed measure on $\mathcal{P}(\Omega)$.

Theorem 1.2. ([8], [9])A concrete logic, $\mathcal{E}$, is regular iff $\operatorname{dim} \mathcal{E}^{\circ}+\operatorname{dim} V(\mathcal{E})=$ $\operatorname{card} \Omega$.

A set $T \subset S_{2}(L)$ is called full if for all $x, y \in L$ it holds $x \leq y \Leftrightarrow \forall f \in$ $T\left(f(x) \leq f(y)\right.$. OMP $L$ is isomorphic to a c.l. iff $S_{2}(L)$ is full. In this case, the sets $x^{\prime}=\left\{\mu \in S_{2}(L) \mid \mu(x)=1\right\}$ form a c.l. $\mathcal{E}=\mathcal{E}(L)$ on $\Omega=S_{2}(L)$ wich is called a total representation of $L$. We also mention a simple criterion of the fullness

\footnotetext{
${ }^{1}$ Department of Mathematics and Mechanics, Kazan State University, Kremlevskaya Street, 18. Kazan, Tatarstan, Russia, 420008.
} 
of a $S_{2}$. The condition equivalent to the fullness of $S_{2}$ is as follows: if $x, y \in A$ are not orthogonal, then there exists $s \in S_{2}(L)$ such that $s(x)=s(y)=1$.

Let us dwell $(3,3)$-hom. finite OMPs, $L$. Let $l_{n}=l\left(P_{0}, \ldots, P_{n-1}, Q_{0}, \ldots, Q_{n-1}\right)$ be a loop [1] of order $n$, where $P_{i}$ denote the atoms of $l_{n}$ lying in the vertices of a $n$-polygon and $Q_{i}$ are the atoms lying in the middles of sides of the $n$-polygon. So $\left\{P_{i}, Q_{i}, P_{i+1}\right\}, i=0, \ldots, n-1$ (indices modulo $n$ ) are all blocks of $l_{n}$. It is easy to see that a two-valued state, $s$, on $l_{n}$ is well determined by $s\left(P_{0}\right), \ldots, s\left(P_{n-1}\right)$. We use the following abbreviation: $P_{01} Q_{0}=\left\{P_{0}, P_{1}, Q_{0}\right\}$.

\section{2. (3,3)-HOMOGENEOUS FINITE OMPs} 16.

Let $L$ be a (3,3)-hom. OMP. Obviously $\operatorname{card} A=\operatorname{card} B \geq 15$ and $\operatorname{card} A \neq$

Theorem 2.1. 1 .If card $A=15$ then $L$ is isomorphic to $\mathcal{L}_{2}^{3}$.

2. There exist (3,3)-hom. OMPs $L_{17}, L_{27}$ with $\operatorname{card} A \in\{17,27\}$. For $L_{17}$ the set $S_{2}=\emptyset$ and $S\left(L_{17}\right)$ is isomorphic to a segment $\left[0 ; \frac{2}{3}\right]$. For $O M P L_{27}$ the set $S_{2}$ is full and total representation of $L_{27}$ is regular.

Proof. 1. Let $\operatorname{card} A=15$. Using Greechie diagram for $L$ it is easy write out all two-valued states of $L$. So, $\operatorname{card} S_{2}=6$ and $S_{2}$ is full. The total representation of $L$ is minimal and isomorphic to $\mathcal{L}_{2}^{3}$.

2. First we construct $L_{17}$. Let us consider loop $l_{7}=l\left(P_{0}, \ldots, P_{6}, Q_{0}, \ldots, Q_{6}\right)$ and add the atoms $R_{0}, R_{1}, R_{2} ; R_{i} \notin l_{7}(i=0,1,2)$. For seven blocks of $l_{7}$ we add following ten blocks:

$$
\begin{gathered}
P_{04} R_{0}, Q_{025}, Q_{03} R_{2}, P_{1} Q_{4} R_{1}, Q_{136}, \\
Q_{15} R_{0}, P_{25} R_{2}, Q_{246}, P_{36} R_{1}, R_{012} .
\end{gathered}
$$

Next we prove that $S\left(L_{17}\right)$ is isomorphic to a segment $\left[0 ; \frac{2}{3}\right]$. Let $s \in S\left(L_{17}\right)$. Put $s\left(P_{0}\right)=x, s\left(Q_{0}\right)=y, s\left(Q_{6}\right)=z, s\left(R_{0}\right)=t$ and $s\left(Q_{2}\right)=u$. We show that all values of $s$ are described by $x$.

1) We have $s\left(P_{1}\right)=1-x-y, s\left(P_{6}\right)=1-x-z, s\left(P_{4}\right)=1-x-t$ and $s\left(Q_{5}\right)=1-u-y, s\left(Q_{4}\right)=1-u-z$. From the blocks $P_{45} Q_{4}, P_{56} Q_{5}$ it follows that $s\left(P_{5}\right)=x+t+u+z-1$ and $t=y$.

2) Now $s\left(Q_{1}\right)=1-s\left(Q_{5}\right)-s\left(R_{0}\right)=u, s\left(P_{2}\right)=x+y-u$ and $s\left(R_{1}\right)=1-s\left(P_{1}\right)-$ $s\left(Q_{4}\right)=x+y+z+u-1$. So $s\left(R_{2}\right)=1-s\left(P_{1}\right)-s\left(Q_{4}\right)=2-x-2 y-u-z$. From the block $P_{25} R_{2}$ we get $u=x$.

3) Next $s\left(R_{1}\right)=1-s\left(P_{1}\right)-s\left(Q_{4}\right)=2 x+y+z-1$. From the blocks $P_{36} R_{1}, Q_{136}$ we calculate $s\left(P_{3}\right)=1-x-y, s\left(Q_{3}\right)=1-x-z$. Then from the block $P_{34} Q_{3}$ we have $1=3-3 x-2 y-z$, or $z=2-2 y-3 x$.

4) If $z$ from 3) is placed to $s\left(R_{2}\right)$ and $s\left(Q_{3}\right)$ then we get $s\left(R_{2}\right)=x, s\left(Q_{3}\right)=$ $2 x+2 y-1$. So from the blocks $Q_{03} R_{2}$ we have $x+y=\frac{2}{3}$.

So the state $s$ has only three values $-x, \frac{2}{3}-x$, and $\frac{1}{3}$, namely:

$x$ - on the atoms $P_{0}, Q_{1}, Q_{2}, R_{2} ;\left(\frac{2}{3}-x\right)$ - on the atoms $P_{2}, Q_{0}, Q_{6}, R_{0}$ $\frac{1}{3}$ - on all other remaining atoms. 
So $S\left(L_{17}\right)$ is isomorphic to the segment $\left[0 ; \frac{2}{3}\right]$

The Greechie diagram of the OMP $L_{27}$ is a cube in three-dimensional space with 3 atoms on each edge, with one atom in center of each side of the cube and with the last atom in the center of cube. The blocks of $L_{27}$ are the lines drawing parallel all axes throw the atoms. Thus, Greechie diagram of $L_{27}$ is divided to three layers. Then state $s \in S_{2}$ is called type 1 (type 2) if $s$ equals 1 on main (secondary) diagonal in one of the layers. Then $S_{2}$ has 6 states type 1 and 6 states type 2 . So, $\operatorname{card} S_{2}=12$. Next $\operatorname{dim} \mathcal{E}^{\circ}=3$ and $\operatorname{dim} V(\mathcal{E})=9$, where $\mathcal{E}$ is the total representation of $L_{27}$. By theorem 1.2. $\mathcal{E}$ is regular.

Theorem 2.2. There exist (3,3)-hom. OMPs with card $A \leq 19$ and with exactly $k$ pure states $(k=1,2, \ldots, 7,10,11)$.

Proof. Let us denote by $H_{k}(m)$ a $(3,3)$-hom. logic with card $A=m$ and $k$ pure states of $S$. Next, we construct a nine OMPs: $H_{1}(19), H_{2}(17), H_{3}(18) H_{4}(19)$, $H_{5}(19), H_{6}(19), H_{7}(18), H_{10}(18), H_{11}(19)$.

We enumerate atoms of $H_{k}(m)$ by a natural numbers $1,2, \ldots, m$ and for a block $\{i, j, n\}$ use abbreviation $i-j-n$. Obviously every such OMP has the following 7 blocks: $B_{1}, \ldots, B_{7}$ : 1-2-3, 1-4-5, 1-6-7, 2-8-9, 2-10-11, 3-12-13, 3-14-15.

1) $H_{1}(19)$. To $B_{1}, \ldots, B_{7}$ we add the following 12 blocks:

4-8-12, 4-10-14, 5-9-16, 5-11-17, 6-8-15, 6-13-16, 7-9-18, 7-14-17, 10-16-19, 11-13-18, 12-17-19, 15-18-19.

From the systen of linear equations $s(i)+s(j)+s(n)=1,\{i, j, n\} \in B$ we found the unique solution $s(i)=\frac{1}{3}(i=1, \ldots, 19)$.

2) $H_{2}(17)$. Consider $L_{17}$ from Theorem 2.1 as $H_{2}(17)$. Then $S$ has two pure states $\left(x=0, x=\frac{2}{3}\right)$.

3) $H_{3}(18)$. To $B_{1}, \ldots, B_{7}$ we add the following 11 blocks:

$4-8-12, \quad 4-14-16,5-10-13,5-17-18,6-11-12,6-16-18$,

$7-9-17,7-10-15,8-15-18,9-13-16,11-14-17$.

Let $s \in S$. Put $s(17)=x, s(18)=y$. Then $s$ has values: $x$ - on the atoms $2,4,6$, 13,$15 ; y$ - on the atoms $1,9,10,12,14 ; 1-x-y$-on the atoms $3,5,7,8,11,16$. The state space $S$ is isomorphic to a triangle: $0 \leq x \leq 1,0 \leq y \leq 1, x+y \leq 1$. So, $S$ has 3 pure states: $(0,0),(1,0),(0,1)$.

4) $H_{4}(19)$. To $B_{1}, \ldots, B_{7}$ we add the following 12 blocks:

$4-8-12,4-10-14,5-9-13,5-11-16,6-8-15,6-11-17$, 7-9-18, 7-10-19, 12-16-18, 13-17-19, 14-17-18, 15-16-19.

Let $s \in S$. Put $s(6)=x, s(8)=y$. Then $s$ has values: $s(1)=2 y-\frac{1}{3}, s(2)=$ $1-2 y, s(3)=s(12)=s(13)=\frac{1}{3}, s(4)=s(5)=\frac{2}{3}-y, s(7)=\frac{4}{3}-x-2 y, s(9)=y$, $s(10)=\frac{2}{3}-x, s(11)=x+2 y-\frac{2}{3}, s(14)=s(18)=x+y-\frac{1}{3}, s(15)=s(16)=$ $1-x-y, s(17)=\frac{5}{3}-2 x-2 y, s(19)=2 x+2 y-1$. 
The state space $S$ is isomorphic to a parallelogram: $0 \leq x \leq \frac{2}{3}, \frac{1}{6} \leq y \leq$ $\frac{1}{2}, \frac{1}{2} \leq x+y \leq \frac{5}{6}$. So, $S$ has 4 pure states:

$$
\left(0, \frac{1}{2}\right),\left(\frac{1}{3}, \frac{1}{6}\right),\left(\frac{2}{3}, \frac{1}{6}\right),\left(\frac{1}{3}, \frac{1}{2}\right) \text {. }
$$

5) $H_{5}(19)$. To $B_{1}, \ldots, B_{7}$ we add the following 12 blocks:

4-8-12, 4-10-14, 5-9-15, 5-11-13, 6-13-16, 6-15-17,

7-11-18, 7-12-19, 8-17-18, 9-16-19, 10-17-19, 14-16-18.

Let $s \in S$. Put $s(15)=x, s(19)=y$. Then $s(18)=y, s(6)=\frac{1}{3}-x+y$ and $s$ has values:

$x$ - on the atoms 1,$13 ;\left(\frac{2}{3}-x\right)$ - on the atoms 3,5 ;

$\left(\frac{2}{3}-y\right)$ - on the atoms $7,16,17 ; \frac{1}{3}-$ on all other remaining atoms.

The state space $S$ is isomorphic to a pentagon: $0 \leq x \leq \frac{2}{3}, 0 \leq y \leq \frac{2}{3}, x-y \leq$ $\frac{1}{3}$. So, $S$ has 5 pure states: $(0,0),\left(0, \frac{2}{3}\right),\left(\frac{1}{3}, 0\right),\left(\frac{2}{3}, \frac{1}{3}\right),\left(\frac{2}{3}, \frac{2}{3}\right)$.

6) $H_{6}(19)$. To $B_{1}, \ldots, B_{7}$ we add the following 12 blocks:

$4-8-12,4-10-14,5-9-13,5-11-16,6-9-15,6-11-17$,

7-10-18, 7-13-19, 8-16-19, 12-17-18, 14-17-19, 15-16-18.

Let $s \in S$. Put $s(2)=x, s(10)=y$. Then $s(5)=x, s(6)=x-y+\frac{1}{3}, s(11)=$ $1-x-y, s(17)=2 y-\frac{1}{3}, s(18)=1-2 y$ and $s$ has also values:

$y$ - on the atoms $7,15,16 ;\left(\frac{2}{3}-x\right)$ - on the atoms 1,9 ;

$\left(\frac{2}{3}-y\right)$ - on the atoms 14,$19 ; \frac{1}{3}-$ on the atoms $3,4,8,12,13$.

The state space $S$ is isomorphic to a hexagon: $0 \leq x \leq \frac{2}{3}, \frac{1}{6} \leq y \leq \frac{1}{2}, y+x \leq$ $1, y-x \leq \frac{1}{3}$. So, $S$ has 6 pure states:

$$
\left(0, \frac{1}{6}\right),\left(0, \frac{1}{3}\right),\left(\frac{1}{6}, \frac{1}{2}\right),\left(\frac{1}{2}, \frac{1}{2}\right),\left(\frac{2}{3}, \frac{1}{3}\right),\left(\frac{2}{3}, \frac{1}{6}\right) .
$$

7) $H_{7}(18)$. To $B_{1}, \ldots, B_{7}$ we add the following 11 blocks:

4-8-12, 4-10-14 5-11-15, 5-16-17, 6-8-18, 6-10-16,

7-9-15, 7-12-17, 9-13-16, 11-13-18, 14-17-18.

Let $s \in S$. Put $s(1)=x, s(3)=y, s(18)=z$. Then $s(2)=1-x-y, s(17)=$ $1-2 z$ and $s$ has also values: $x$ - on the atoms 8,$10 ; y$ - on the atoms 9 , $11 ;(1-x-z)-$ on the atoms 4,$6 ; z-$ on the atoms $5,7,12,14,16 ;(1-y-z)$ - on the atoms 13,15 .

The state space $S$ is isomorphic to a polytope in three-dimensional space: $0 \leq x \leq 1,0 \leq y \leq 1,0 \leq z \leq \frac{1}{2}, x+y \leq 1, x+z \leq 1, y+z \leq 1$. So, $S$ has 7 pure states:

$(0,0,0),(0,1,0),(1,0,0),\left(0,0, \frac{1}{2}\right),\left(0, \frac{1}{2}, \frac{1}{2}\right),\left(\frac{1}{2}, 0, \frac{1}{2}\right),\left(\frac{1}{2}, \frac{1}{2}, \frac{1}{2}\right)$.

8) $H_{10}(18)$. To $B_{1}, \ldots, B_{7}$ we add the following 11 blocks:

$4-8-12,4-10-14$ 5-8-16, 5-11-17, 6-12-16, 6-14-17, 7-9-13, 7-11-15, 9-17-18, 10-13-18, 15-16-18.

Let $s \in S$. Put $s(6)=x, s(16)=y, s(17)=z$. Then $s(1)=2 y-x, s(11)=$ $2 y-z, s(12)=1-x-y, s(14)=1-x-z, s(11)=1-y-z$ and $s$ has also values: $x$ - on the atoms 3,$4 ; y$ - on the atoms $8,9,13 ;(1-2 y)$ - on the atoms $2,5,7$; 
$z$ - on the atoms 5,$10 ;(1-y-z)$ - on the atoms 13,15 .

The state space $S$ is isomorphic to a polytope in three-dimensional space: $0 \leq x \leq \frac{2}{3}, 0 \leq y \leq \frac{1}{2}, 0 \leq z \leq \frac{2}{3}, x+y \leq 1, x+z \leq 1, y+z \leq 1, \frac{x}{2} \leq y, \frac{z}{2} \leq y$.

So, $S$ has 10 pure states:

$$
\begin{aligned}
& (0,0,0),\left(0, \frac{1}{2}, 0\right),\left(\frac{1}{2}, \frac{1}{2}, 0\right),\left(\frac{1}{2}, \frac{1}{2}, \frac{1}{2}\right),\left(0, \frac{1}{2}, \frac{1}{2}\right), \\
& \left(0, \frac{1}{3}, \frac{2}{3}\right),\left(\frac{1}{3}, \frac{1}{3}, \frac{2}{3}\right),\left(\frac{1}{2}, \frac{1}{4}, \frac{1}{2}\right),\left(\frac{2}{3}, \frac{1}{3}, \frac{1}{3}\right),\left(\frac{2}{3}, \frac{1}{3}, 0\right) .
\end{aligned}
$$

9) $H_{11}(19)$. To $B_{1}, \ldots, B_{7}$ we add the following 12 blocks:

4-8-12, 4-10-14 5-9-15, 5-11-13, 6-8-16, 6-10-17,

7-13-19, 7-15-18, 9-17-19, 11-16-18, 12-17-18, 14-16-19.

Let $s \in S$. Put $s(10)=x, s(15)=y, s(19)=z$. Then $s(1)=x+y-\frac{1}{3}, s(6)=$ $\frac{1}{3}-x+z, s(7)=1-y-z$ and $s$ has also values:

$x$ - on the atom $8 ; y$ - on the atom $13 ; z$ - on the atom 18 ;

$\frac{2}{3}-x$ - on the atoms 2,$4 ; \frac{2}{3}-y$ - on the atoms 3,$5 ; \frac{2}{3}-z-$ on the atoms 16,17 ;

$\frac{2}{3}-z$ - on the atoms 16,$17 ; \frac{1}{3}$-on the atoms $9,11,12,14$.

The state space $S$ is isomorphic to a polytope in three-dimensional space: $0 \leq x \leq \frac{2}{3}, 0 \leq y \leq \frac{2}{3}, 0 \leq z \leq \frac{2}{3}, x+y \geq \frac{1}{3}, z \geq x-\frac{1}{3} 1, y+z \leq 1$.

So, $S$ has 11 pure states:

$$
\begin{gathered}
\left(\frac{1}{3}, 0,0\right),\left(0, \frac{1}{3}, 0\right),\left(0, \frac{2}{3}, 0\right),\left(\frac{1}{3}, \frac{2}{3}, 0\right),\left(\frac{2}{3}, \frac{2}{3}, \frac{1}{3}\right),\left(\frac{2}{3}, 0, \frac{1}{3}\right), \\
\left(0, \frac{2}{3}, \frac{1}{3}\right),\left(\frac{1}{3}, 0, \frac{2}{3}\right),\left(\frac{2}{3}, 0, \frac{2}{3}\right),\left(\frac{2}{3}, \frac{1}{3}, \frac{2}{3}\right),\left(0, \frac{1}{3}, \frac{2}{3}\right) .
\end{gathered}
$$

Remark 2.3. The special interest have (3,3)-hom. OMPs with a unique state. What least number of atoms of such logic? An example with 22 atoms till now was known [2]. The example, constructed by us, has 19 atoms, and we did not manage to construct OMP with smaller number of atoms. Probably number of atoms 19 cannot be reduced; we yet have no the proof it. In [11] was is developed a method of construction OMPs with a unique state, however these of logics are not homogeneous.

Using Greechie loop lemma [1] it is not difficult to show, that all listed above (3,3)-hom. logic $H_{k}(m)$ are not orthomodular lattices. Certainly the examples of orthomodular lattices with such property will have the much greater number of atoms.

Remark 2.4. There is a well-known method of constructing the finite $(3,3)$ hom. OMPs with even $\operatorname{card} A$. Let $n \geq 9$ and $A=\left\{a_{i} \mid i=0,1, \ldots, 2 n-1\right\}$ be a set of atoms. Then sets $\left\{a_{2 i}, a_{2 i+1}, a_{2 i+2}\right\} ;\left\{a_{2 i-5}, a_{2 i}, a_{2 i+5}\right\}$ (indices modulo $2 \mathrm{n})$ as the blocks generate some $(3,3)$-hom. logic $L(2 n)$. For example, $L(22)$ has one, $L(20)$ has two, and $L(18)$ has three pure states for the corresponding state spaces. But the author is not familiar with any convenient method of constructing such OMPs with odd $\operatorname{card} A$.

Remark 2.5. (3,3)-hom. logics arise when we consider a relational OMPs [4] on a finite set. For example, the relational OMP on 8 -element set is $(3,3)$ - 
homogeneous. Every horizontal summand of this OMP has 28 atoms and exactly one pure state. We present all blocks of this summand:

1-13-15, 1-6-21, 1-14-22, 2-6-25, 2-5-17, 2-18-26, 3-9-13,

3-10-25, 3-14-26, 4-9-17, 4-10-21, 4-18-22, 5-15-19, 6-23-27,

$7-11-13,7-12-25,7-15-27,8-11-21,8-12-17,8-19-23,9-16-20$,

10-24-28, 11-16-24, 12-20-28, 14-24-27, 15-20-26, 16-19-22, 18-23-28.

\section{ACKNOWLEDGMENTS.}

This reseach was supported by RFBR (grant no. 01-01-00129) and URFR (grant no. UR.04.01.061).

\section{REFERENCES}

1. Kalmbach, G., Orthomodular Lattices, Academic Press, London, (1983).

2. Greechie, R.J, Miller, F.R., On structures related to states on an empirical logic I. Weights on finite spaces, Technical Report 16, Dept. of Math., Kansas State Univ., Manhattan, Kansas, (1970), 1-25.

3. Kohler, E., Orthomodulare Verbande mit Regularitatsbedingungen (Orthomodular lattices with regularity conditions), Journal of Geometry, Vol. 119 (1982), 129-145 (in German).

4. Harding, J. Decompositions in quantum logic, Trans. Amer.Math. Soc., 348 (1996), 1839-1862.

5. Navara, M., and Rogalewicz, V., Constructions of orthomodular lattices with given state spaces, Demostratio Math., 21 (1988), 481-493.

6. Navara, M., and Tkadlec, J., Automorphisms of concrete logics, Comment. Math. Univ. Carolinae Math., 32 (1991), 15-25.

7. Navara, M., An orthomodular lattice admiting no group-valued measure, Proc. Amer.Math. Soc.,122(1994), 7-12.

8. Ovchinnikov, P.G. and Sultanbekov, F.F. Finite concrete logics: their structure and measures on them, Int. J. Theor.Phys., 37(1998), 147-153.

9. Ovchinnikov, P.,Measures on finite concrete logics, Proc. Amer.Math. Soc.,127 (1999), 1957-1966.

10. Ovchinnikov, P.G., On homogeneous finite Greechie logics admiting a two-valued state, In: Teor. funktsii, prilozh. i sm. vopr., Kazan State University, Kazan, (1999), 167-168 (Russian).

11. Ptak, P.,Exotic logics, Coll. Math. 56 (1987), 1-7.

12. Rogalewicz, V. A remark on $\lambda$-regular orthomodular lattices, Aplikace Mat., 34 (1989), 449-452.

13. Sultanbekov, F.F. Signed measures and automorphisms of a class of finite concrete logics, Konstr. Teoriy funktsii i Funk Analiz (Kazan), 8(1992), 57-68 (in Russian).

Mail: Department of Mathematics and Mechanics, Kazan State University, Kremlevskaja Street, 18. Kazan, Tatarstan, Russia, 420008.

E-mail: Foat.Sultanbekov@ksu.ru 